\title{
Optimization of $40 \mathrm{~Gb} / \mathrm{s}$ WDM Systems Using Super-Gaussian RZ Pulses
}

\author{
Jong-Hyung Lee*, Dae-Hyun Han, and Yong-Jae Lee \\ Department of Electronic Engineering, Dongeui University, Busan, 614-714, Korea \\ Byeong-Yoon Choi \\ Department of Computer Engineering, Dongeui University, Busan, 614-714, Korea
}

(Received September 26, 2008 : revised October 30, 2008 : accepted November 11, 2008)

\begin{abstract}
40 Gbps WDM Systems using super-Gaussian RZ pulses have been studied by numerical simulation to optimize their performance. The assumption of standard single mode fiber is valid when existing WDM systems are required to upgrade their performance to $40 \mathrm{Gbps}$. It is shown that the standard single mode fiber can transmit optical signals over $720 \mathrm{~km}(\mathrm{Q}>10)$ by optimizing optical and electrical filter characteristics at the receiver and by compensation of dispersion. However, it is also shown that $\pm 0.3 \%$ dispersion compensation tolerance per span $(80 \mathrm{~km})$ could prohibit transmitting over $320 \mathrm{~km}(\mathrm{Q}>10)$. In addition, a duty cycle of less than 0.4 degrades system performance significantly.
\end{abstract}

Keywords: RZ format, Standard Single Mode Fiber, WDM, 40Gb/s

OCIS codes : (060.0060) Fiber optics and optical communications; (060.2330) Fiber optics communications; (060.4510) Optical communications

\section{INTRODUCTION}

As the demand for bandwidth is ever increasing, we often need to decide how to upgrade the transmission capacity in installed WDM systems. One of the easiest ways is to increase bit rate of each channel, which requires no replacement of the installed fibers. However, most installed fiber, which is the standard single mode fiber (SSMF), has large chromatic dispersion, typically $16 \mathrm{ps} /$ $\mathrm{nm} / \mathrm{km}$ at $1550 \mathrm{~nm}$ window. Therefore, the system performance using SSMF can be degraded significantly at a higher bit rate if cautious design methodology does not apply[1-3].

In this paper, we investigate by numerical simulations the optimum performance of $40 \mathrm{Gbps}$ WDM systems using the standard single mode fiber. In section II, the receiver performance is optimized first without fibers using RZ format with a duty cycle of 0.5 . In section III, transmission performance is investigated using the optimum receiver in section II. Chromatic dispersion is assumed to be compensated $100 \%$ by the commercially

*Corresponding author: jonghlee@deu.ac.kr available dispersion compensation fiber, DCF. Section IV shows the simulation results with decreased RZ duty cycles to find the possible improvements, and simulation results including residual dispersion and transmitter chirp are discussed in section V. Conclusions follow in section VI.

\section{OPTIMIZATION OF THE RECEIVER PERFORMANCE}

To achieve maximum Q-values[4,5], we first consider a $40 \mathrm{~Gb} / \mathrm{s}$ WDM system using RZ format without fibers to optimize the optical and electrical receiver bandwidths. Figure 1 shows the spectrum and the eye diagram (channel @ $193.41 \mathrm{THz}$ ) of the RZ transmitter with a duty cycle of 0.5 , which was used to evaluate $40 \mathrm{~Gb} / \mathrm{s}$ WDM transmission over dispersion compensated standard SMF. To reduce simulation time, only a 5 channel WDM signal was investigated, with the center channel @ $193.41 \mathrm{THz}$ chosen to obtain an estimate of system performance.

The bandwidths of the optical filters and the electrical filters were varied independently in the range of $30 \cdots 110$ 


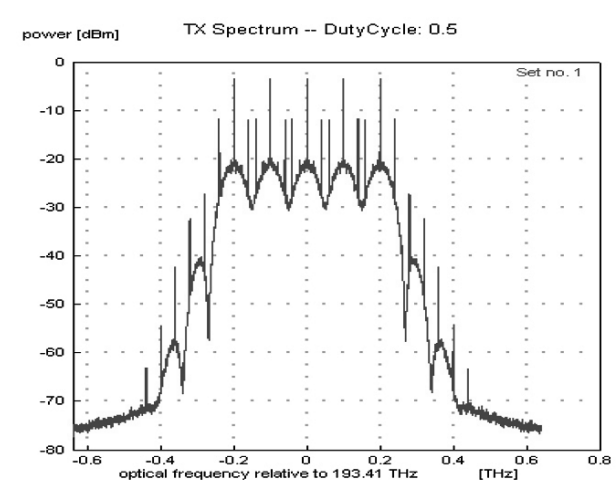

(a)

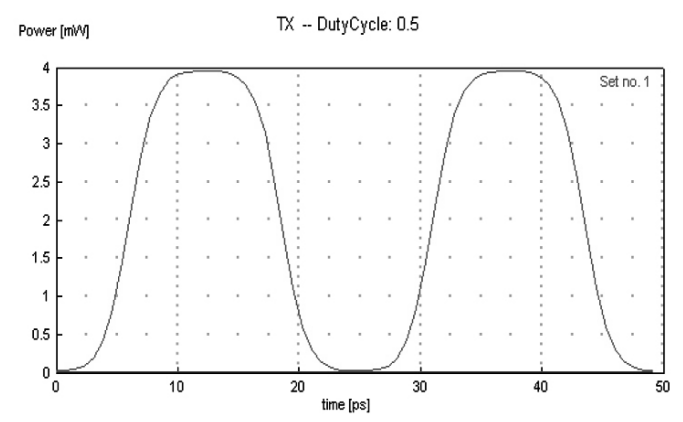

(b)

FIG. 1. Spectrum (a) and eye diagram (b) of the 40 $\mathrm{Gb} / \mathrm{s}$ RZ Transmitter with a duty cycle of 0.5

$\mathrm{GHz}$ and $20 \cdots 70 \mathrm{GHz}$, respectively. To emulate a realistic scenario regarding the noise disturbances at the receiver, the noise source was included right after the transmitter to give an optical signal-to-noise ratio $\mathrm{OSNR}_{\mathrm{Tx}}=20$ $\mathrm{dB}$ (in a measurement bandwidth of $100 \mathrm{GHz}$ ). Figure 2 shows the results for a Sinc-shaped and a Gaussianshaped optical receiver filter [6]. In both cases, the electrical receiver filter as a post-detection filter was a 5 th order Bessel low-pass. In the figure solid lines show the Q-values using the optimum bandwidth of the post-detection filter. We could obtain a larger optimum Q-value with the Gaussian optical filter. Because the Gaussian-shaped optical filter performs better than the Sinc-shaped filter, a $101 \mathrm{GHz}$ Gaussian optical filter and a 5th order Bessel filter with a cutoff frequency of $53 \mathrm{GHz}$, which are optimum, were used for all simulations with a duty cycle of 0.5 .

\section{TRANSMISSION RESULTS WITH A RZ DUTY CYCLE OF 0.5}

In this section, we investigate the transmission effects of the 5 channel WDM system with the optimum receiver bandwidths found in section II. Figure 3 shows the repeated transmission path for the performance evaluation of 40

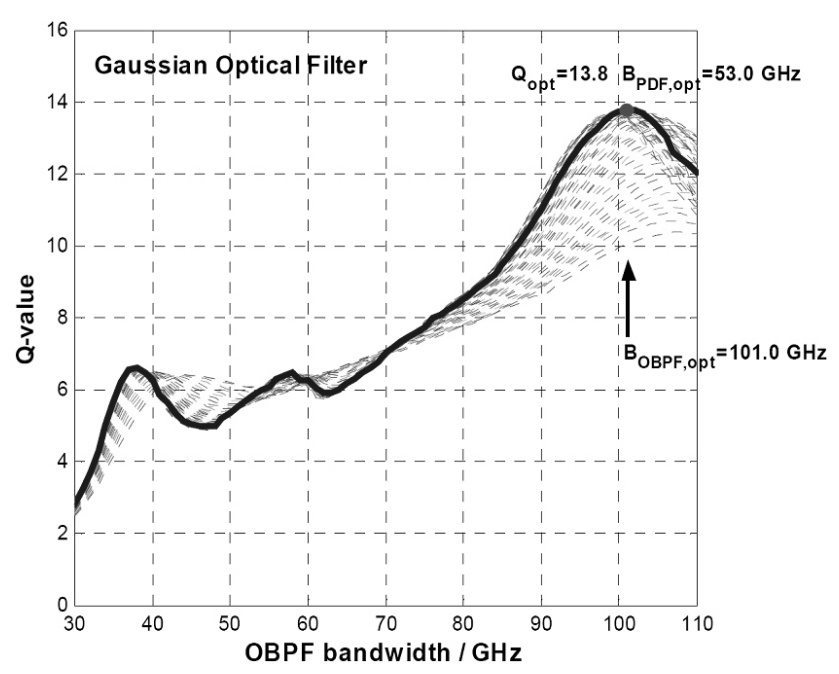

(a)

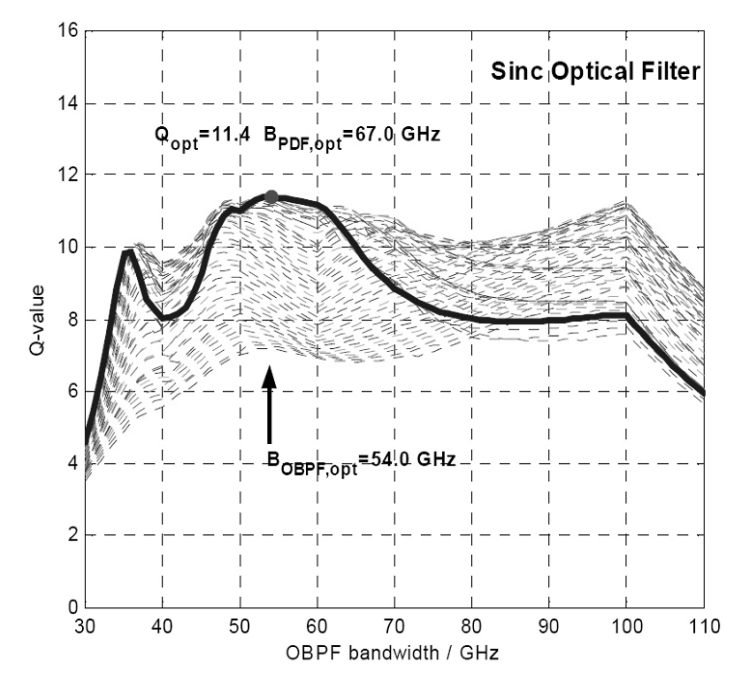

(b)

FIG. 2. Q-values vs. optical filter bandwidth (a) Gaussian filter (b) Sinc-shaped filter

$\mathrm{Gb} / \mathrm{s}$ RZ transmission. EDFA is assumed to have flat gain characteristics with $6 \mathrm{~dB}$ noise figure and the fiber parameters used in simulation are included in Table 1. One span consists of $80 \mathrm{~km} \mathrm{SSMF,} \mathrm{DCF,} \mathrm{and} \mathrm{two} \mathrm{EDFAs}$ to compensate fiber losses. The chromatic dispersion is post-compensated by DCF, and the input power into the $\operatorname{SSMF}(-4.0 \ldots+6.0 \mathrm{dBm})$ and into the $\operatorname{DCF}(-6.0$ $\cdots+4.0 \mathrm{dBm})$ were varied. For the central channel at $193.41 \mathrm{THz}$, the Q-value was monitored after each span. The transmission distance after each loop is considered as the length of SSMF, which is set to $80 \mathrm{~km}$. A chirp free transmitter signal and a compensation ratio of $100 \%$ were assumed.

Figure 4 shows the calculated Q-values vs. SSMF input power per channel for different transmission distances in simulations. For each Q-value, the optimum launch power per channel into the DCF is shown (small numbers 
TABLE 1. Fiber Parameters

\begin{tabular}{c|c|c|c|c|c}
\hline \hline & $\begin{array}{c}\text { Attenuation } \\
{[\mathrm{dB} / \mathrm{km}]}\end{array}$ & $\begin{array}{c}\text { Chromatic Dispersion } \\
{[\mathrm{ps} / \mathrm{nm} / \mathrm{km}]}\end{array}$ & $\begin{array}{c}\text { Dispersion Slope } \\
{\left[\mathrm{ps} / \mathrm{nm}^{2} / \mathrm{km}\right]}\end{array}$ & $\begin{array}{c}\text { Effective Core Area } \\
{\left[\mathrm{\mu m}^{2}\right]}\end{array}$ & $\begin{array}{c}\text { Nonlinear Refractive } \\
\text { Index }\left[\mathrm{m}^{2} / \mathrm{W}\right]\end{array}$ \\
\hline $\mathrm{SSMF}$ & 0.23 & 16 & 0.08 & 80 & $2.6 \times 10^{-20}$ \\
\hline $\mathrm{DCF}$ & 0.5 & -90 & $-0.08 \times 90 / 16$ & 50 & $2.6 \times 10^{-20}$ \\
\hline
\end{tabular}

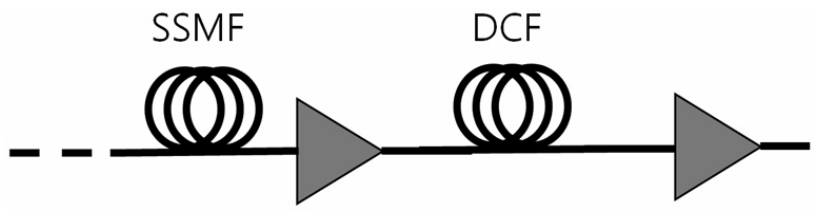

FIG. 3. Repeated transmission path

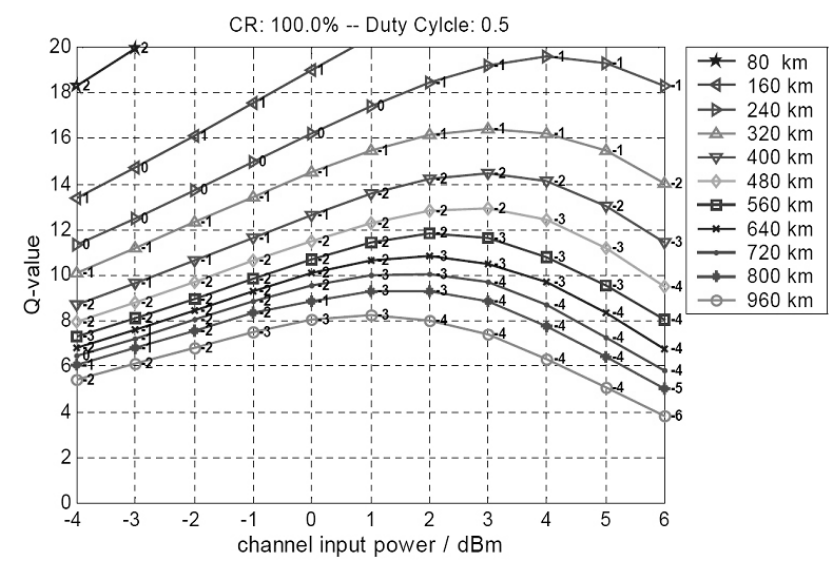

FIG. 4. Q-values vs. SSMF launch power per channel after different transmission distances.

next to the symbols, in $\mathrm{dBm}$ ). As can be seen in Figure 4, transmission distances of up to $720 \mathrm{~km}$ (9 spans) can be bridged for $\mathrm{Q} \geq 10$. In this case, the optimum launch power per channel for the SSMF and the DCF are 2 $\mathrm{dBm}$ and $-3 \mathrm{dBm}$, respectively. The corresponding contour plot is shown in Figure 5. Figure 5 indicates the sensitivity of the maximum $\mathrm{Q}$ value in terms of launch power per channel for the SSMF and the DCF.

\section{RZ TRANSMISSION FOR DECEASED RZ DUTY CYCLES}

\section{Optimization of the receiver performance}

To improve system performance, we analyzed the aforementioned 5-channel RZ-system with modified duty cycles (0.4 and 0.3) of the transmitted RZ waveform[7]. Again, initially the receiver filters were optimized with respect to the particular duty cycle. Since the central channel is supposed to be most affected by its neighbors, only this channel was analyzed as before. Additionally, only the Gaussian optical receiver filter was considered, since it always performs better than its Sinc-shaped

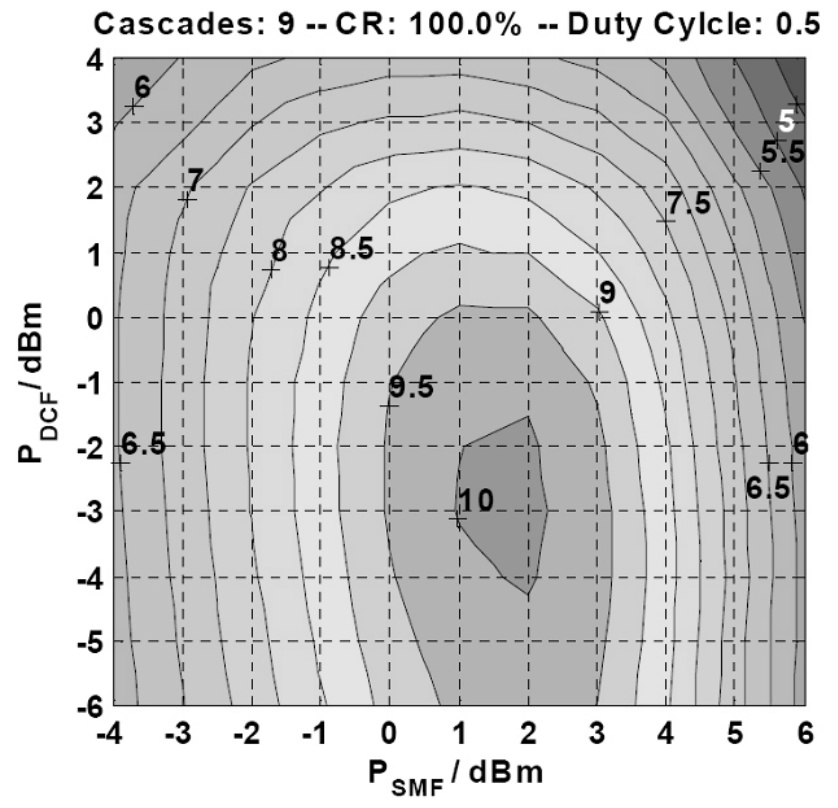

FIG. 5. Q-values vs. launch powers per channel after 9 spans.

counterpart in section II.

For the investigated duty cycles of 0.4 and 0.3 , Figure 6 shows the resultant Q-values vs. the optical and electrical receiver bandwidths. The optimum $\mathrm{Q}$ value for a duty cycles of 0.4 was obtained with a Gaussian-shaped optical filter $\left(\mathrm{B}_{\mathrm{OBPF}, \mathrm{opt}}=89 \mathrm{GHz}\right)$ and a 5 th order electrical Bessel low-pass filter $\left(\mathrm{B}_{\mathrm{PDF}}\right.$, opt $\left.=37 \mathrm{GHz}\right)$ in the receiver.

On the other hand, for a duty cycle of 0.3 we observed a severe breakdown of the system performance $\left(\mathrm{Q}_{\mathrm{opt}}=\right.$ 6.8). This results from an excessive spectral broadening, which is inherently associated with the decrease of the duty cycle. As a result, in this case the system performance is supposed to be limited mainly by inter-channel crosstalk rather than by noise or by signal distortions due to SPM-GVD interactions.

Thus, simulations with Super-Gaussian pulses for duty cycles smaller than 0.4 are not promising and consequently are not considered further.

\section{Results of RZ transmission with a RZ duty cycle of 0.4}

The results of the system simulations for the postcompensated systems are depicted in Figure 7 and Figure 8. A chirp free transmitter signal with a RZ duty cycle 


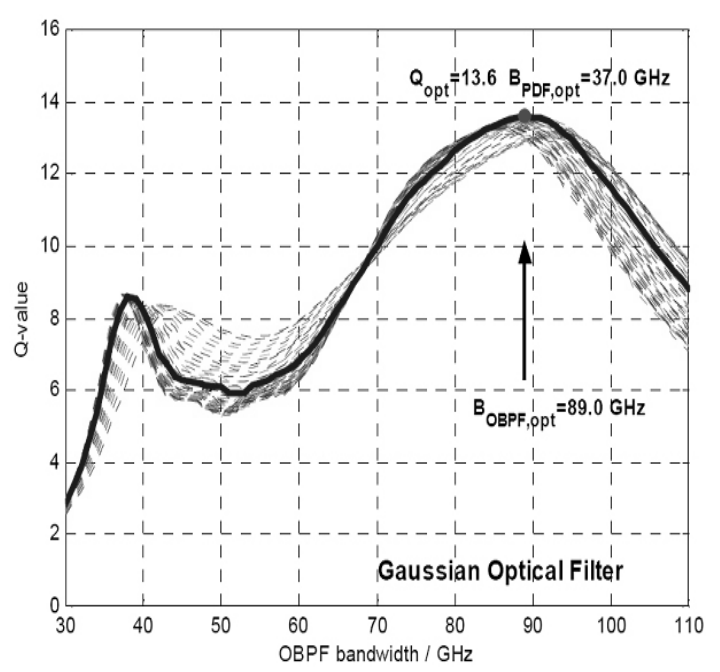

(a)

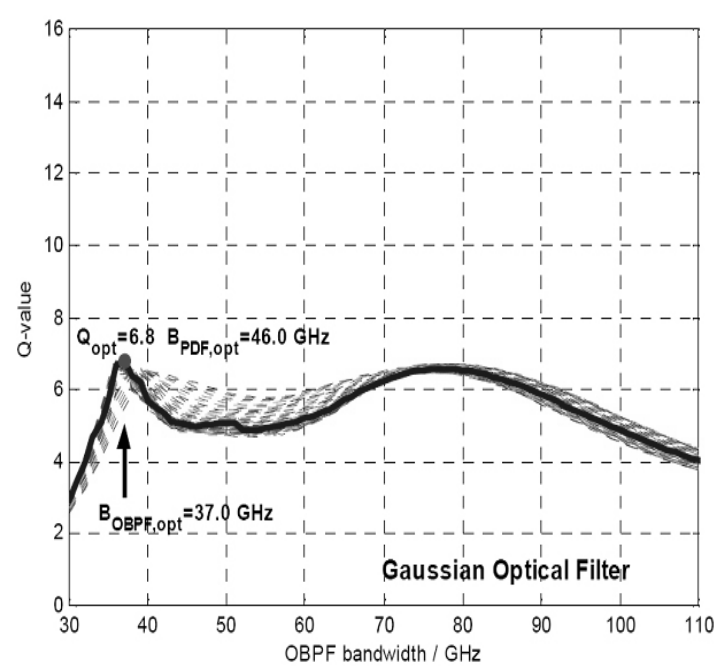

(b)

FIG. 6. Q-values vs. optical filter bandwidth (a) duty cycle $=0.4(\mathrm{~b})$ duty cycle $=0.3$

of 0.4 and a compensation ratio of chromatic dispersion $=100 \%$ were assumed. As in the previous simulations, the input powers into the SSMF $(-4.0 \cdots+6.0 \mathrm{dBm})$ and into the DCF $(-6.0 \cdots+4.0 \mathrm{dBm})$ were varied independently to identify the optimum operating condition.

With the post-compensation of chromatic dispersion, distances of up to $720 \mathrm{~km}$ (9 spans) can be bridged for $\mathrm{Q} \geq 10$ ( Figure 7 ). The optimum input powers into the SSMF and the DCF are about $2 \mathrm{dBm}$ and $-2 \mathrm{dBm}$, respectively. The corresponding contour plot is shown in Figure 8. Compared to the results obtained for a RZ duty cycle of 0.5 (Figure 4 and Figure 5), no significant change (actually a slight increase of the Q-values) can be observed for the RZ transmission with a duty cycle of 0.4 .

It's worth noting that the observed similarity of the results for RZ duty cycles 0.5 and 0.4 is only found with

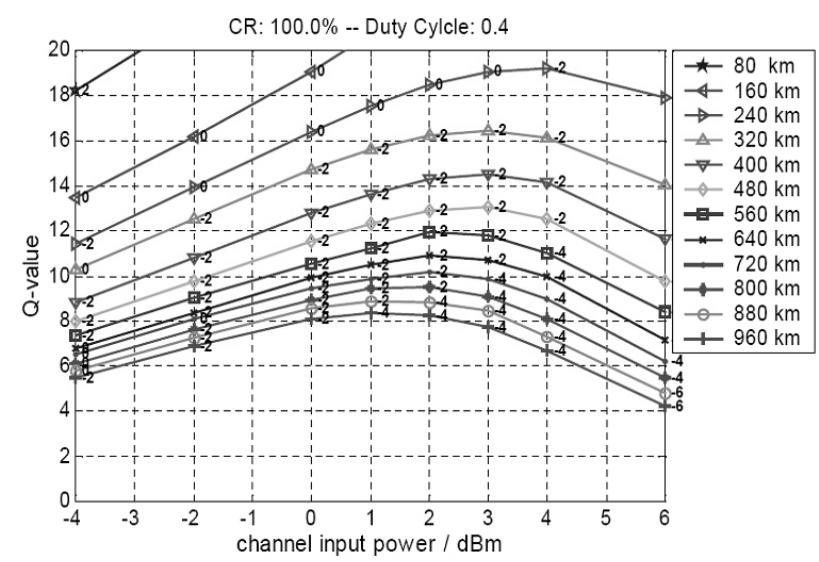

FIG. 7. Q-values vs. SSMF launch power per channel after different transmission distances with a duty cycle of 0.4 .

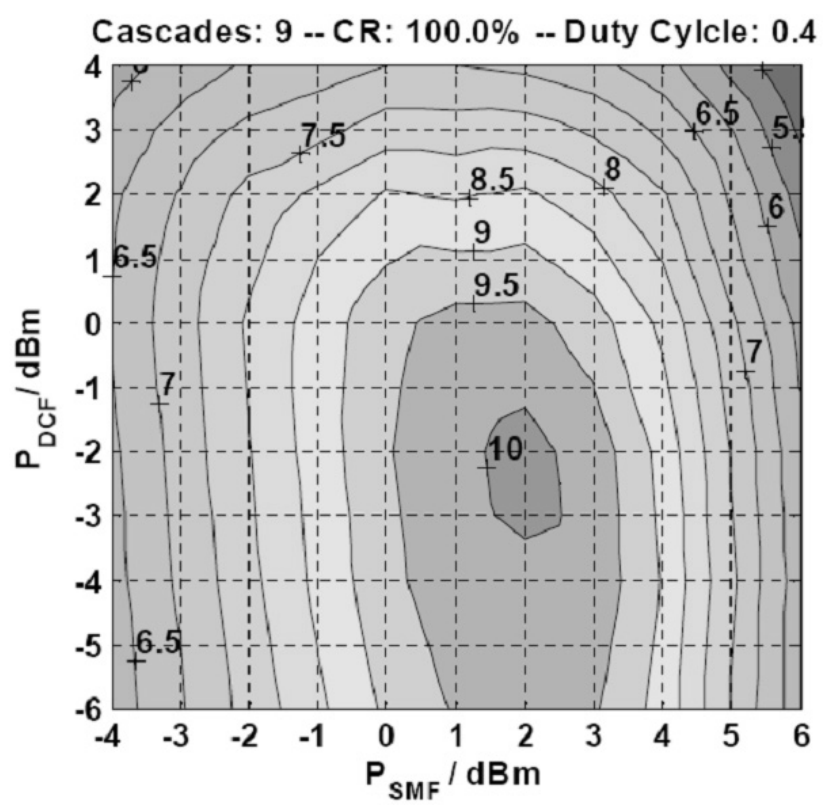

FIG. 8. Q-values vs. launch powers per channel after 9 spans with a duty cycle of 0.4 .

the individually optimized receiver filters. Practically, that means even a slight change in the transmitted pulse shape requires an adjustment of the respective receiver filters.

\section{EFFECTS OF RESIDUAL CHROMATIC DISPERSION AND TRANSMITTER CHIRP}

Since the dispersion parameter D is wavelengthdependent, $100 \%$ compensation may not be achievable due to the dispersion slope mismatch between SSMF and DCF if the same DCF must compensate dispersion over the entire bandwidth of a WDM system. To study the effects of residual dispersion, the dispersion value 


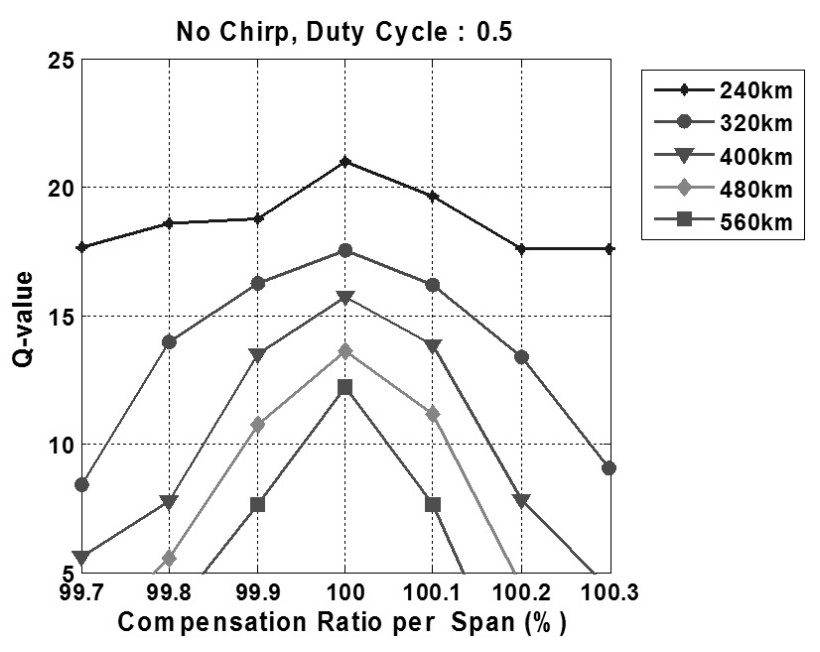

FIG. 9. Q-values vs. compensation ratio per span.

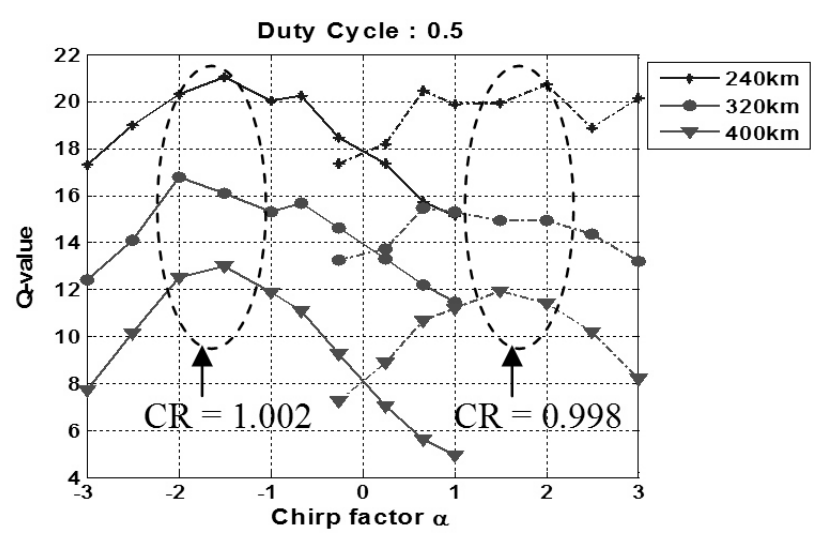

(a)

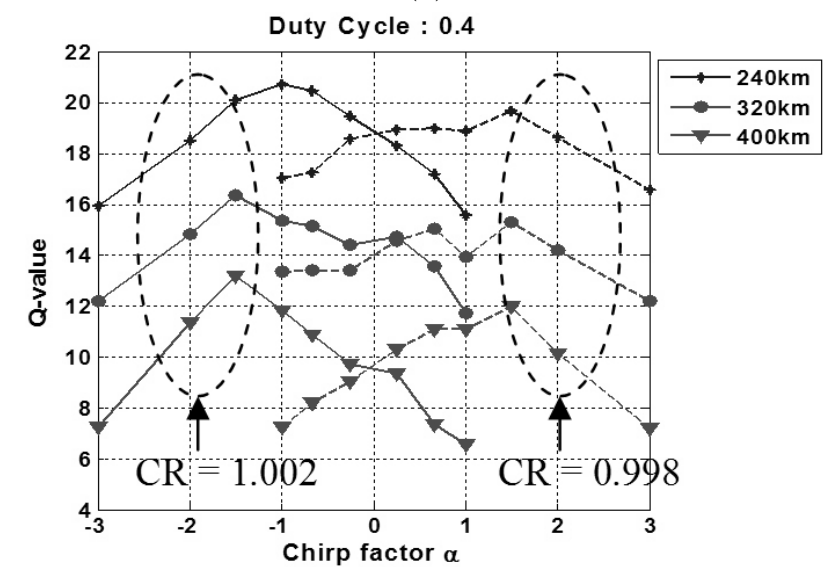

(b)

FIG. 10. Effects of transmitter chirp factor (a) duty cycle $=0.5(\mathrm{~b})$ duty cycle $=0.4$

of DCF has been varied slightly from $99.7 \%$ (undercompensated) to $100.3 \%$ (over-compensated). All other simulation parameters were the same as in section III with the optimized signal power levels. While transmission distance is increased, the accumulated residual dispersion is indeed more detrimental to the system performance as observed in Fig. 9. Unless a dispersion slope compensator is employed, $\pm 0.3 \%$ dispersion compensation tolerance per span could prohibit transmitting over 320 $\mathrm{km}(\mathrm{Q}>10)$.

To verify the possibly positive influence of modulator chirp combined with residual dispersion on system performance, the chirp of the Mach-Zehnder modulator (MZM) in simulations was varied. The chirp behavior of the MZM is typically characterized by the chirp factor $\alpha$ [8-10]. While the effect of the modulator chirp is not noticeable with $100 \%$ compensation ratio, Fig. 10 suggests that we could obtain significant improvements in system performance by controlling the chirp in the presence of residual dispersion even though careful selection of chirp sign is required for each channel.

\section{CONCLUSION}

In this paper, we studied 40 Gbps WDM systems numerically to find optimum parameters. It is assumed that optical fiber is standard single mode fiber, which is most widely installed in the world, and the transmitter pulse is super-Gaussian RZ.

We optimized the bandwidth of the filters at the receiver(optical and electrical), and showed that Gaussianshaped optical filter in the receiver can have better performance compared to Sinc-shaped filter. We also showed that the duty cycle of less than 0.4 in the transmitter pulse will degrade system performance due to its wider spectrum. In addition, by optimizing input power levels to SSMF and DCF, we showed that we may achieve that over $720 \mathrm{~km}(\mathrm{Q}>10)$ transmission distance if dispersion is $100 \%$ compensated. The effect of residual dispersion and transmitter chirp is also investigated. In $40 \mathrm{~Gb} / \mathrm{s}$ systems using installed fibers, PMD, which is not included in this study, could be a critical system impairment. It is known that RZ performs better than NRZ in the presence of PMD and a smaller duty cycle is more resistent to PMD $[11,12]$. However, the effect of broader spectra due to shorter pulses on dispersion and inter-channel interferences is not clear yet. By designing system with a higher margin, we may avoid system failure due to PMD effects. For optimum system design we need to further investigate PMD effects includ ing the impact of duty cycle, which will be one of the future topics.

\section{ACKNOWLEDGMENT}

This work was supported by Dong-eui University Grant 2008AA169. 


\section{REFERENCES}

[1] H. Taga, "Long distance transmission experiments using WDM technology,” J. Lightwave. Tech., vol. 14, no. 6, pp. 1287-1298, June 1996.

[2] D. Breuer, K. Obermann, K. Petermann, "Comparison of $\mathrm{N} \times 40 \mathrm{~Gb} / \mathrm{s}$ and $4 \mathrm{~N} \times 10 \mathrm{~Gb} / \mathrm{s}$ WDM Transmission over standard single-mode fiber at $1.55 \mu \mathrm{m}$, , IEEE Photon. Technol. Lett., vol. 10, no. 12, pp. 1793-1795, Dec. 1998.

[3] F. Forghieri, "Granularity in WDM Networks: The Role of Fiber Nonlinearities," IEEE Photon. Technol. Lett., vol. 8, no. 10, pp. 1400-1402, Oct. 1996.

[4] C.J. Anderson, J.A. Lyle, "Technique for evaluating system performance using $\mathrm{Q}$ in numerical simulations exhibiting intersymbol interference," Electronics Letters, vol. 30, no. 1, pp. 71-72, Jan. 1994.

[5] G.P. Agrawal, Lightwave Technology Telecommunication Systems (John Wiley \& Sons, New Jersey, 2005) Chapter 5.

[6] C. K. Madsen and J. H. Zhao, Optical Filter Design and Analysis, (John Wiley \& Sons, New York, 1999) Chapter 4.
[7] M.I. Hayee, A.E. Willner, "NRZ Versus RZ in 10-40 Gb/s Dispersion-Managed WDM Transmission Systems," IEEE Photon. Technol. Lett., vol. 11, no. 8, pp. 991-993, 1999.

[8] G. Wenke, M. Kimmek, "Considerations on the $\alpha$-factor of Nonideal, External Optical Mach-Zehnder Modulators," J. Opt. Comm., vol. 17, no. 2, p. 42-48, 1996.

[9] F. Koyama, K. Iga, "Frequency Chirping in external Modulators," J. Lightwave. Tech., vol. 6, no. 1, pp. 8793, Jan. 1988.

[10] A. H. Gnauck, R.W. Tkach, M. Mazurczyk, "Interplay of Chirp and Self Phase Modulation in Dispersion-Limited Optical Transmission Systems," Proceedings of European Conference on Optical Communications, TuC4.4, pp. 105-108, 1993.

[11] H. Sunnerud, M. Karlsson, and P. A. Andrekson, "Analytical theory for PMD-compensation," IEEE Photon. Technol. Lett., vol. 12, pp. 50-52, Jan. 2000.

[12] H. Sunnerud, M. Karlsson, and P. A. Andrekson, "A comparison between NRZ and RZ Data Formats with respect to PMD-Induced System Degradation," IEEE Photon. Technol. Lett., vol. 13, no. 5, pp. 448- 450, May 2001. 